\title{
Prospective analysis of the association of infection with CagA bearing strains of Helicobacter pylori and coronary heart disease
}

\author{
R K Singh, A D McMahon, H Patel, C J Packard, B J Rathbone, N J Samani for the West \\ of Scotland Coronary Prevention Group
}

Heart 2002;88:43-46

See end of article for authors' affiliations

Correspondence to: Prof N J Samani, Department of Cardiology, Clinical Sciences Wing, Glenfield General Hospital, Groby Road, Leicester LE3 9QP, UK; nis@le.ac.uk

Accepted 13 March 2002

\begin{abstract}
Objective: To see whether it was possible to replicate in a prospective study the association recently reported between infection with the more virulent (type 1) cytotoxin associated gene A (CagA) antigen carrying strains of Helicobacter pylori and increased risk of coronary heart disease.

Design and setting: Nested case-control study in a clinical outcomes trial.

Subjects: Participants in the West of Scotland coronary prevention study.

Methods: $H$ pylori CagA serological status was determined in plasma samples of 201 subjects (cases) who subsequently had a coronary event during follow up and in 414 subjects (controls) matched for age and smoking who remained event-free, using a semiquantitative commercial enzyme linked immunosorbent assay (ELISA) kit against the p120 antigen of CagA.

Results: $105(52 \%)$ in the case group and $176(43 \%)$ in the control group were seropositive lodds ratio (OR) $1.49,95 \%$ confidence interval $(\mathrm{CI}) 1.06$ to $2.10, \mathrm{p}=0.022)$. The association remained significant after adjustment for blood pressure, body mass index, plasma concentrations of low density lipoprotein and high density lipoprotein cholesterol, history of hypertension and diabetes, statin treatment, and socioeconomic status (OR $1.51,95 \% \mathrm{Cl} 1.06$ to 2.16, $\mathrm{p}=0.023)$. Baseline inflammatory markers (white cell count, $\mathrm{C}$ reactive protein, fibrinogen) were not significantly increased in either $\mathrm{H}$ pylori CagA positive cases or controls.

Conclusions: The findings provide support from a prospective study for the hypothesis that there is an association between infection with CagA bearing strains of $H$ pylori and coronary heart disease. The mechanism(s) underlying the association remain to be elucidated.
\end{abstract}

$\mathrm{T}$ here is increasing evidence from both clinical and experimental observations that inflammation plays an important part in the pathogenesis of coronary heart disease (CHD). ${ }^{12}$ Systemic markers of inflammation such as $\mathrm{C}$ reactive protein and acute phase reactants such as fibrinogen and serum amyloid A have been shown to be associated with risk of myocardial infarction. ${ }^{3-6}$ A potential cause of the inflammation leading to CHD is chronic infection, and in the past decade a large number of studies have tried to find a link between the two. ${ }^{7}$

A particular focus of attention has been Helicobacter pylori. $H$ pylori is a microaerophilic spiral shaped Gram negative bacterium that colonises the gastric lumen of humans and other primates. Infection is commonly acquired in childhood and is usually chronic. ${ }^{8}$ The bacterium is now recognised to be of major aetiological importance in peptic ulcer disease ${ }^{9}$ and in gastric cancer. ${ }^{10}$ Mendall and colleagues ${ }^{11}$ were the first group to report a higher prevalence of $H$ pylori seropositivity in patients with CHD than in healthy volunteers. However, subsequent studies have produced conflicting findings ${ }^{7}$ and the significance of the association remains uncertain. Confounding by the relation of $H$ pylori infection to other CHD risk factors such as age and social class may, at least partly, explain the contradictory results.

However, recent studies have explored another possibility. There is genetic diversity between $H$ pylori strains, which affects virulence. ${ }^{12}$ Specifically, strains bearing the cytotoxin associated gene A (CagA) provoke a heightened inflammatory response in vivo $^{13}$ and show a stronger relation with peptic ulcer disease ${ }^{14}$ and gastric cancer. ${ }^{15}$ In 88 patients with CHD (age 57 (8) years, 74 men) and in 88 matched controls, Pasceri and colleagues ${ }^{16}$ observed a 3.8 -fold adjusted increase in risk of CHD in H pylori CagA seropositive subjects. More recently, we reported that in subjects $<65$ years old ( 153 patients recruited at the time of their myocardial infarction, 153 controls), $H$ pylori CagA seropositivity was associated with a 1.8-fold increase in risk of myocardial infarction, which increased further to 2.25 -fold in subjects $<55$ years old. ${ }^{17}$ These findings require confirmation in further studies. Here we report a prospective analysis of the association between seropositivity for CagA bearing strains of $H$ pylori and CHD in subjects in the West of Scotland coronary prevention study (WOSCOPS).

\section{METHODS}

\section{Study design and subjects}

In WOSCOPS, 6595 men under the age of 65 years who had low density lipoprotein (LDL) cholesterol concentrations between $4.5-6.0 \mathrm{mmol} / \mathrm{l}$, but who had no history of a myocardial infarction, were randomly assigned to receive $40 \mathrm{mg}$ of pravastatin or placebo daily. ${ }^{18}$ Over a mean follow up period of 4.9 years, the incidence of the primary end point, a composite of non-fatal myocardial infarction and death from CHD, was $31 \%$ lower with pravastatin treatment. ${ }^{18}$ Risk reductions of the same magnitude were seen for revascularisation procedures (coronary artery bypass and percutaneous transluminal

Abbreviations: $\mathrm{CagA}$, cytotoxin associated gene $\mathrm{A} ; \mathrm{CHD}$, coronary heart disease; $\mathrm{Cl}$, confidence interval; ELISA, enzyme linked immunosorbent assay; HDL, high density lipoprotein; LDL, low density lipoprotein; MCDS, mean Carstairs deprivation score; OR, odds ratio; WOSCOPS, West of Scotland coronary prevention study 
Table 1 Baseline characteristics of case and control groups

\begin{tabular}{llll}
\hline & Case group $(\mathrm{n}=201)$ & Control group $(\mathrm{n}=414)$ & $\mathrm{p}$ Value \\
\hline Age (years) * & $56.9(5.3)$ & $56.9(5.3)$ & 0.956 \\
Smokers $(\%)^{*}$ & 51.2 & 52.2 & 0.829 \\
Body mass index $\left(\mathrm{kg} / \mathrm{m}^{2}\right)$ & $25.7(3.1)$ & $25.8(3.0)$ & 0.865 \\
Systolic blood pressure (mm Hg) & $140.6(18.5)$ & $137.1(17.4)$ & 0.024 \\
Diastolic blood pressure (mm Hg) & $86.5(11.1)$ & $84.1(10.8)$ & 0.012 \\
$\mathrm{LDL}$ cholesterol (mmol/l) & $5.0(0.4)$ & $4.9(0.4)$ & 0.412 \\
$\mathrm{HDL}$ cholesterol (mmol/I) & $1.1(0.2)$ & $1.1(0.2)$ & 0.234 \\
Diabetes (\%) & 1.5 & 1.2 & 0.773 \\
Hypertension (\%) & 25.4 & 15.0 & 0.002 \\
Statin treatment (\%) & 44.8 & 47.6 & 0.513 \\
\hline \multirow{2}{*}{ *Age and smoking were matching criteria. For quantitative variables mean (SD) is given. HDL, high density } \\
lipoprotein; LDL, low density lipoprotein.
\end{tabular}

coronary angioplasty). In total 580 subjects had a primary end point (507) or a revascularisation (77) as a first event. ${ }^{18}$ For the current analysis a nested case-control approach was adopted. Plasma samples were available from the one year follow up visit. In the first year, 118 events occurred. Among subjects who were event-free at this point, 214 (46\%) who subsequently developed an event (177 primary end point, 24 revascularisations) were randomly chosen. Each was matched with two subjects who remained event-free, for a total of 428 controls, on the basis of age (using two year age categories), duration of follow up, and smoking status, with subjects categorised as either current smokers or non-smokers/former smokers.

\section{Measurements}

All major risk factors were assessed during recruitment. ${ }^{19}$ Fasting total, LDL, and high density lipoprotein (HDL) cholesterol concentrations were measured. Haemtaological variables including the white cell count were determined and fibrinogen was assayed by heat precipitation nephelometry. ${ }^{19} \mathrm{C}$ reactive protein was measured more recently in plasma collected at recruitment and stored at $-70^{\circ} \mathrm{C}$ using a high sensitivity, two site enzyme linked immunoassay. ${ }^{20}$ Socioeconomic status was assessed using the mean Carstairs deprivation score (MCDS). ${ }^{21}$ Education level (categories: none, school, further, or university) and employment status (categories: employed, unemployed, retired, or invalid) were recorded.

IgG antibodies to the CagA protein were quantified in plasma samples using a commercial enzyme linked immunosorbent assay (ELISA) kit (Helicobacter pl20 (CAGA) ELISA, Viva Diagnostics, Hurth, Germany). We have previously validated the ELISA by concurrent western blotting analysis to confirm the presence or absence of CagA antibodies using a reference strain (NCTC 11637, National Collection of Type Cultures, London, UK) as antigen. ${ }^{17}$ A cut off value of $>7.5 \mathrm{U}$ was taken to categorise samples as positive as recommended by the manufacturer. Laboratory staff were blinded to the case-control status of the samples.

\section{Statistical analysis}

Quantitative variables were compared between the case and control groups, and between $H$ pylori CagA positive and $H$ pylori CagA negative subjects using analysis of variance. Qualitative variables were compared using $\chi^{2}$ test. Conditional logistic regression analysis was used to assess the independent association of H pylori CagA seropositivity with risk of CHD. Adjustment was made for CHD risk factors and socioeconomic status as assessed by the MCDS. Quantitative variables were included as continuous variables and qualitative variables as categorical variables. Odds ratios (OR) and 95\% confidence intervals (CI) were calculated. Correlation of $\mathrm{H}$ pylori CagA seropositivity and MCDS was assessed using the Spearman rank correlation.
Table 2 Cytotoxin associated gene A (CagA) serological status in case and control groups

\begin{tabular}{lll}
\hline & Case group & Control group \\
\hline CagA seropositive & $105(52 \%)$ & $176(42 \%)$ \\
CagA seronegative & $96(48 \%)$ & $238(58 \%)$ \\
\hline
\end{tabular}

There is a significant association of $\mathrm{CagA}$ seropositivity and development of coronary heart disease $(p=0.022)$.

\section{RESULTS}

Plasma samples for $H$ pylori CagA analysis were available for 201 (94\%) of the chosen cases and $414(98 \%)$ of the controls. Table 1 summarises the clinical characteristics of these subjects. The groups did not differ in most parameters except that systolic and diastolic blood pressures were higher and frequency of hypertension greater in the case group. Interestingly, in these randomly selected subgroups, plasma lipid concentrations were not different between the case and control groups and randomisation to statin treatment was similar.

Table 2 summarises $H$ pylori CagA serological status for the case and control groups. H pylori CagA seropositivity and subsequent development of CHD were significantly associated (OR $1.49,95 \%$ CI 1.06 to $2.10, \mathrm{p}=0.022$ ). The association remained significant after adjustment for blood pressure, body mass index, plasma concentrations of LDL and HDL cholesterol, history of hypertension and diabetes, and pravastatin treatment (OR 1.49,95\%CI 1.05 to $2.12, \mathrm{p}=0.026$ ). There was a significant correlation of $H$ pylori CagA seropositivity with the MCDS $(r=0.114, \mathrm{p}=0.020)$, although it was not significantly associated with CHD. Nevertheless, inclusion of the MCDS in the multivariate conditional logistic regression analysis did not significantly alter the relation of $H$ pylori CagA seropositivity with development of CHD (OR 1.51, 95\% CI 1.06 to $2.16, p=0.023$ ). We also explored the effect of individual components of socioeconomic status. There was a significant negative association between CagA seropositivity and education level attained in both the case and control groups, but no relation with employment status (table 3 ). However, neither education level (OR 1.58, 95\% CI 1.07 to $2.15, \mathrm{p}=0.019$ ) nor employment status (OR $1.53,95 \%$ CI 1.08 to $2.16, \mathrm{p}=0.017$ ) had an effect on the association of CagA seropositivity with development of CHD. Similarly, there was no effect of pravastatin treatment (OR $1.72,95 \%$ CI 1.02 to 2.88 in pravastatin treated subjects; OR $1.33,95 \%$ CI 0.84 to 2.12 in placebo treated subjects, test for interaction $\mathrm{p}=0.672$ ). Finally, the association remained significant when subjects with revascularisation were omitted and analysis was restricted to the 177 subjects with the primary end point of non-fatal myocardial infarction or death from CHD (OR 1.44, 95\% CI 1.01 to 2.05 , $\mathrm{p}=0.047)$.

To investigate whether $H$ pylori CagA seropositivity was associated with evidence of increased systemic inflammation, 
Table 3 Comparison of levels of risk factors and inflammatory markers by CagA status in case and control groups

\begin{tabular}{|c|c|c|c|c|c|c|}
\hline & \multicolumn{3}{|l|}{ Case group } & \multicolumn{3}{|l|}{ Control group } \\
\hline & CagA positive $(n=105)$ & CagA negative ( $n=96)$ & $p$ Value & CagA positive $(n=176)$ & CagA negative $(n=238)$ & p Value \\
\hline Age (years) & $57.6(5.1)$ & $56.1(5.4)$ & 0.039 & $57.2(5.1)$ & $56.6(5.5)$ & 0.203 \\
\hline Smokers (\%) & 51.5 & 51.0 & 0.956 & 57.9 & 47.9 & 0.043 \\
\hline BMI $\left(\mathrm{kg} / \mathrm{m}^{2}\right)$ & $26.0(3.2)$ & $25.5(2.9)$ & 0.217 & $25.7(2.8)$ & $25.8(3.1)$ & 0.716 \\
\hline LDL cholesterol (mmol/l) & $5.0(0.4)$ & $5.0(0.4)$ & 0.998 & $5.0(0.4)$ & $4.9(0.4)$ & 0.461 \\
\hline HDL cholesterol (mmol/l) & $1.1(0.2)$ & $1.1(0.3)$ & 0.140 & $1.1(0.2)$ & $1.1(0.2)$ & 0.452 \\
\hline SBP $(\mathrm{mm} \mathrm{Hg})$ & $139(18)$ & $142(19)$ & 0.359 & $137(17)$ & $137(17)$ & 0.671 \\
\hline $\mathrm{DBP}(\mathrm{mm} \mathrm{Hg})$ & $86(10)$ & 87 (12) & 0.254 & 84 (11) & 84 (11) & 0.873 \\
\hline Diabetes (\%) & 0.0 & 3.1 & 0.068 & 1.1 & 1.3 & 0.905 \\
\hline Hypertension (\%) & 27.6 & 22.9 & 0.444 & 15.9 & 14.4 & 0.660 \\
\hline MCDS & $1.34(3.48)$ & $0.40(3.50)$ & 0.060 & $1.55(3.72)$ & $0.33(3.50)$ & $<0.001$ \\
\hline Further education $(\%)^{*}$ & 16.1 & 28.1 & 0.041 & 13.1 & 24.9 & 0.003 \\
\hline Employment $(\%) \dagger$ & 60.0 & 64.6 & 0.504 & 59.7 & 65.8 & 0.199 \\
\hline WCC $\left(\times 10^{-3} / \mathrm{mm}^{3}\right)$ & $7.0(2.0)$ & $7.0(2.1)$ & 0.947 & $6.9(1.9)$ & $6.6(1.8)$ & 0.141 \\
\hline Fibrinogen (g/l) & $4.4(0.8)$ & $4.5(1.0)$ & 0.347 & $4.4(1.1)$ & $4.4(0.9)$ & 0.581 \\
\hline $\operatorname{CRP}(\mathrm{mg} / \mathrm{l}) \ddagger$ & $4.1(4.9)$ & $4.6(6.6)$ & 0.567 & $3.7(7.7)$ & $3.2(5.3)$ & 0.715 \\
\hline
\end{tabular}

we compared baseline values for white cell count, serum fibrinogen, and $\mathrm{C}$ reactive protein in the case and control groups stratified by CagA status. Table 3 shows these data, together with values for the classic risk factors. None of the inflammatory markers were significantly increased in either $H$ pylori CagA positive patients or controls.

\section{DISCUSSION}

In this study, we provide evidence from a prospective study that seropositivity for the CagA strains of $H$ pylori is an independent risk marker for the development of clinical CHD in middle aged white men. We adopted a nested case-control approach to test our hypothesis that $H$ pylori CagA seropositivity would confer increased risk. On the basis of previous estimates of a prevalence of CagA seropositivity in white populations (approximately 40\%), ${ }^{14-17}$ our study was sufficiently powered $(>90 \%)$ to detect the previously reported 1.8 -fold increase in risk in subjects who were $H$ pylori CagA seropositive. ${ }^{17}$ As our study population was derived from a cardiovascular clinical trial, detailed and accurate information was available at baseline on other cardiovascular risk factors ${ }^{19}$ to permit adequate adjustments to be made for potential confounding factors.

Apart from the initial study of Pasceri and colleagues ${ }^{16}$ and our own previous case-control study of patients recruited at the time of their acute event, ${ }^{17}$ to our knowledge only two other studies and only one other prospective study have investigated the association of $H$ pylori CagA seropositivity and CHD. In a case-control study of 312 patients with stable CHD and 479 controls recruited from healthy blood donors, Koenig and colleagues ${ }^{22}$ found an OR for CHD given a positive $H$ pylori CagA status of 1.5 (95\% CI 1.0 to 2.1 ), which decreased to 1.1 (95\% CI 0.7 to 1.7 ) after adjustment for smoking, diabetes, hypertension, alcohol consumption, number of years of formal education, and plasma HDL cholesterol. More recently, Whincup and associates ${ }^{23}$ reported findings from the prospective British regional heart study. In 505 subjects who developed CHD over a mean follow up of 16 years and 1025 age matched controls who remained symptom-free, the OR for CHD for subjects who were $H$ pylori CagA seropositive at baseline was 1.42 (95\% CI 1.06 to 1.91 ), which fell to 1.10 (95\% CI 0.71 to 1.71) after adjustment for risk factors. The decrease was particularly pronounced and the OR became insignificant after adjustments for markers of adult and childhood socioeconomic status. ${ }^{23}$
Lack of information on and therefore adjustment for confounders has been proposed as an important reason for the variable associations reported between $H$ pylori infection and CHD. ${ }^{7}$ In particular, many studies have not adjusted for socioeconomic status, which is known to affect both risk of CHD and $H$ pylori carrier status. As illustrated by the excellent study of Whincup and associates, ${ }^{23}$ often when socioeconomic status is taken into account, the observed association between $H$ pylori seropositivity and CHD is decreased or lost. However, the appropriateness of such adjustment needs some discussion. How socioeconomic deprivation increases risk of CHD is not fully known. Increased susceptibility to chronic $H$ pylori infection may provide one mechanism. If this is the case, then $H$ pylori CagA seropositivity becomes an intermediate step in the causal pathway and adjustment for socioeconomic status is likely, by definition, to reduce any association between infection and CHD risk. In this study, we observed a significant correlation between $H$ pylori CagA seropositivity and the MCDS, a validated instrument for measuring socioeconomic status. ${ }^{21}$ H pylori CagA seropositivity was also associated with a significantly higher MCDS in controls and a borderline significantly higher score in the case group (table 3 ). However, adjustment for the MCDS or for two other individual markers of socioeconomic status (education level and employment status) did not influence the relation between $H$ pylori CagA seropositivity and CHD in the WOSCOPS subjects. This suggests that the relation between infection with $H$ pylori CagA strains and risk of CHD is not entirely explained by socioeconomic status, although a limitation of our study is the lack of information on detailed childhood socioeconomic status.

Initial studies suggested that $H$ pylori seropositivity is associated with evidence of increased systemic inflammation, ${ }^{24} 25$ thus providing a possible mechanistic link between chronic infection and CHD. However, a recent meta-analysis ${ }^{26}$ suggested that the correlations were caused by chance or publication bias. As in previous studies, ${ }^{3-6}$ baseline plasma C reactive protein concentrations, white cell count, and serum fibrinogen were strong predictors of the risk of coronary events in WOSCOPS. ${ }^{20}$ However, we found no evidence of increased values of these markers in subjects who were $H$ pylori CagA seropositive. A potential limitation of our study is that the inflammatory markers and CagA serological status were not measured on samples collected at the same time. The markers were measured from samples collected at the time of recruitment into WOSCOPS, whereas CagA antibodies were measured from samples collected at one year (that is, the 
baseline for this study; see Methods). However, since $H$ pylori infection is usually acquired in childhood or in early adult life, ${ }^{8}$ it is unlikely that there was a significant shift in serological status during the first year of WOSCOPS to obscure any relation. Further, our findings are similar to those observed by Koenig and colleagues ${ }^{22}$ and Whincup and associates. ${ }^{23}$ Another potential mechanism is through direct infection of vascular tissue. However, in contrast to other infectious agents such as Chlamydia pneumoniae, $H$ pylori has not been consistently isolated from atherosclerotic tissue, ${ }^{27}{ }^{28}$ although a recent study ${ }^{29}$ has reported finding $H$ pylori DNA in 20 of 38 carotid plaques and morphological and immunohistochemical evidence of $H$ pylori infection in half the DNA positive plaques. Therefore, the mechanism by which infection with $H$ pylori CagA bearing strains predisposes to CHD remains unclear.

Although the increased risk associated with $H$ pylori CagA seropositivity is relatively small, it has a high prevalence in over $40 \%$ of the population. It is therefore possible that the attributable risk is considerable, especially if the recent concept that the risk of CHD associated with infections may be multiplicative and related to the "pathogen burden" is substantiated.$^{30}$ Strategies aimed at infection prevention and eradication, which have already been successfully used to reduce the risk of peptic ulcer disease, may have a considerable impact on the incidence of CHD. However, before randomised trials of antibiotics against $H$ pylori for the prevention of CHD can be recommended, further data from prospective studies are necessary given the conflicting findings so far.

In summary, we report evidence from a prospective study of an independent and moderate association of seropositivity for CagA bearing strains of $H$ pylori and risk of subsequent CHD, at least in middle aged white men.

\section{Authors' affiliations}

R K Singh, H Patel, N J Samani, Division of Cardiology, Department of Medicine, University of Leicester, Leicester, UK

A D McMahon, Robertson Centre for Biostatistics, Glasgow University, Glasgow, UK

C J Packard, Department of Pathological Biochemistry, Glasgow Royal Infirmary, Glasgow, UK

\section{REFERENCES}

1 Ross R. The pathogenesis of atherosclerosis: a perspective for the 1990s. Nature 1993;362:801-8.

2 Ridker PM. Inflammation, infection, and cardiovascular risk: how good is the clinical evidence? Circulation 1998:97:1671-4

3 Liuzzo G, Biasucci LM, Gallimore JR, et al. The prognostic value of C-reactive protein and serum amyloid $A$ protein in severe unstable angina. N Engl J Med 1994;331:417-24.

4 Kuller LH, Tracy RP, Shaten J, et al, for the MRFIT Research Group. Relationship of C-reactive protein and coronary heart disease in the MRFIT nested case-control study. Am J Epidemiol 1996;144:537-47.

5 Haverkate F, Thompson SG, Pyke SDM, et al. Production of C-reactive protein and risk of coronary events in stable and unstable angina. Lancet 1997;349:462-6.

6 Ridker PM, Cushman M, Stampfer M, et al. Inflammation, aspirin, and the risk of cardiovascular disease in apparently health men. N Engl J Med 1997;336:973-9.
7 Danesh J, Collins R, Peto R. Chronic infections and coronary heart disease: is there a link? Lancet 1997;350:430-6.

8 Graham DY, Malaty HM, Evans GE, et al. Epidemiology of Helicobacter pylori in an asymptomatic population in the United States. Gastroenterology 1991;100:1495-501.

9 Kurata JH, Nogawa AN. Meta-analysis of risk factors for peptic ulcer: nonsteroidal anti-inflammatory drugs, Helicobacter pylori, and smoking. $J$ Clin Gastroenterol 1997;24:2-17.

10 Blaser MJ, Chyou PH, Nomura A. Age at establishment of Helicobacter pylori infection and gastric carcinoma, gastric ulcer, and duodenal ulcer risk. Cancer Res 1995;55:562-5.

11 Mendall MA, Goggin PM, Molineaux N, et al. Relation of Helicobacter pylori infection and coronary heart disease. Br Heart J 1994:71:437-9.

12 Atherton JC. H. pylori virulence factors. Br Med Bull 1998;54:105-20.

13 Peek RM Jr, Miller GG, Tham KT, et al. Heightened inflammatory response and cytokine expression in vivo to cagA+ Helicobacter pylori strains. Lab Invest 1995:73:760-70.

14 Ching CK, Wong BC, Kwok E, et al. Prevalence of cagA-bearing Helicobacter pylori strains detected by the anti-cagA assay in patients with peptic ulcer disease and in controls. Am J Gastroenterol 1996;91:949-53.

15 Blaser MJ, Perez-Perez Gl, Kleanthous $\mathrm{H}$, et al. Infection with Helicobacter pylori strains possessing cagA is associated with an increased risk of developing adenocarcinoma of the stomach. Cancer Res 1995:55:2111-5.

16 Pasceri V, Cammarota G, Patti G, et al. Association of virulent Helicobacter pylori strains with ischemic heart disease. Circulation 1998;97: 1675-9.

17 Gunn M, Stephens JC, Thompson JR, et al. Significant association of CagA positive Helicobacter pylori strains with risk of premature myocardial infarction. Heart 2000;84:267-71

18 Shepherd J, Cobbe SM, Ford I, et al. Prevention of coronary heart disease with pravastatin in men with hypercholesterolemia: the west of Scotland coronary prevention study. N Engl J Med 1995;333:1301-7.

19 The WOSCOPS Study Group. Screening experience and baseline characteristics in the west of Scotland coronary prevention study. Am J Cardiol 1995:76:485-91.

20 Packard CJ, O'Reilly DS, Caslake M, et al. Lipoprotein-associated phospholipase A2 as an independent predictor of coronary heart disease. N Engl J Med 2000;343:1 148-55.

21 McLoone P, Boddy FA. Deprivation and mortality in Scotland, 1981 and 1991. BM 1994:309:1465-70.

22 Koenig W, Rothenbacher D, Hoffmeister A, et al. Infection with Helicobacter pylori is not a major independent risk factor for stable coronary heart disease: lack of a role of cytotoxin-associated protein A-positive strains and absence of a systemic inflammatory response. Circulation 1999;100:2326-31.

23 Whincup P, Danesh J, Walker $M$, et al. Prospective analysis of potentially virulent strains of Helicobacter pylori and coronary heart disease in middle-aged men. Circulation 2000;101:1647-52.

24 Patel P, Carrington D, Strachan DP, et al. Fibrinogen: a link between chronic infection and coronary heart disease. Lancet 1994;343: 1634-5.

25 Mendall MA, Patel P, Ballam L, et al. C reactive protein and its relation to cardiovascular risk factors: a population based cross-sectional study. BMU 1996:312:1061-5.

26 Danesh J, Peto R. Risk factors for coronary heart disease and infection with Helicobacter pylori: meta-analysis of 18 studies. BM 1998:316:1130-2.

27 Blasi F, Ranzi ML, Erba M, et al. No evidence for the presence of Helicobacter pylori in atherosclerotic plaques in abdominal aortic aneurysm specimens. Atherosclerosis 1996;126:339-40.

28 Radke PW, Merkelbach-Bruse S, Messmer BJ, et al. Infectious agents in coronary lesions obtained by endatherectomy: pattern of distribution coinfection, and clinical findings. Coron Artery Dis $2001 ; 12: 1-6$.

29 Ameriso SF, Fridman EA, Leiguarda RC, et al. Detection of Helicobacter pylori in human carotid atherosclerotic plaques. Stroke 2001:32:385-91.

30 Zhu J, Nieto FJ, Horne BD, et al. Prospective study of pathogen burden and risk of myocardial infarction or death. Circulation 2001;103:45-51. 